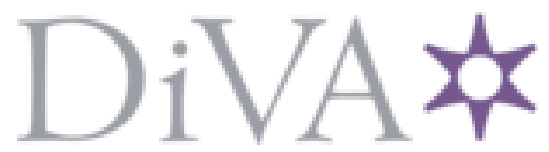

http://www.diva-portal.org

\title{
Postprint
}

This is the accepted version of a paper published in Ecology Letters. This paper has been peerreviewed but does not include the final publisher proof-corrections or journal pagination.

Citation for the original published paper (version of record):

Kazancioglu, E., Arnqvist, G. (2014)

The maintenance of mitochondrial genetic variation by negative frequency-dependent selection.

Ecology Letters, 17(1): 22-27

https://doi.org/10.1111/ele.12195

Access to the published version may require subscription.

N.B. When citing this work, cite the original published paper.

Permanent link to this version:

http://urn.kb.se/resolve?urn=urn:nbn:se:uu:diva-216052 


\section{The maintenance of mitochondrial genetic variation by negative frequency-dependent selection}

\section{Erem Kazancıŏlu and Göran Arnqvist*}

Animal Ecology, Department of Ecology and Genetics, Uppsala University, Norbyv. 18 D, SE-752 36 Uppsala, Sweden.

${ }^{*}$ Correspondence: E-mail: goran.arnqvist@ebc.uu.se

Author contributions: G.A. conceived the research; E.K. and G.A. designed and performed the experiment, analyzed the data and wrote the manuscript.

Running head: Frequency-dependent selection on mtDNA

Key words: mtDNA, population biology, habitat heterogeneity, niche, competition, phylogeography, epistasis, life history evolution, Callosobruchus.

Number of words in the abstract: 148

Number of words in the main text: 3007

Number of references: 50

Number of figures: 2

Number of tables: 1 


\begin{abstract}
Mitochondrial genes generally show high levels of standing genetic variation, which is puzzling given the accumulating evidence for phenotypic effects of mitochondrial genetic variation. Negative frequency-dependent selection, where the relative fitness of a genotype is inversely related to its frequency in a population, provides a potent and potentially general process that can maintain mitochondrial polymorphism. We assessed the change in mitochondrial haplotype frequencies over 10 generations of experimental evolution in 180 seed beetle populations in the laboratory, where haplotypes competed for propagation to subsequent generations. We found that haplotypes consistently increased in frequency when they were initially rare and decreased in frequency when initially common. Our results have important implications for the use of mtDNA haplotype frequency data to infer population level processes and they revive the general hypothesis that negative frequency-dependent selection, presumably caused by habitat heterogeneity, may commonly promote polymorphism in ecologically relevant life history genes.
\end{abstract}




\section{INTRODUCTION}

The processes that maintain ecological and genetic diversity are not fully understood (Chesson 2000). In particular, explaining the maintenance of genetic variation in the face of natural selection is one of the most longstanding and outstanding problems in biology (Lewontin 1974; Charlesworth \& Hughes 2000). Briefly, the problem is that natural selection should drive alleles that encode high fitness to fixation while deleterious alleles should be lost, resulting in the erosion of genetic variation. Yet, fitness and ecologically relevant life history characters typically show much higher levels of standing genetic variation than expected under mutation-selection balance (Charlesworth \& Hughes 2000).

The general problem of understanding the maintenance of genetic variation is exacerbated for the small but vital part of the eukaryotic genome that is carried within the mitochondria (i.e., mtDNA), because the mitochondrial genome shows maternal (i.e., cytoplasmic) inheritance, has a relatively low effective population size, is haploid and does generally not recombine. The mitochondrial genome encodes key building blocks of the cellular energy producing pathway (Ballard \& Melvin 2010). Mitochondrial DNA has been extensively used for a long time in population biology and ecology as a genetic marker, under the implicit or explicit assumption that alternative extant mtDNA alleles are functionally equivalent and thus selectively neutral. However, it has gradually become evident that the pattern of molecular variation in mtDNA is often inconsistent with a neutral expectation (e.g., Nachman et al. 1996; Ruiz-Pesini et al. 2004; Ballard \& Rand 2005; Bazin et al. 2006; Breen et al. 2012; Frankham 2012) and mtDNA haplotype frequencies have been found to correlate with environmental factors in natural populations (e.g., Grant et al. 2006). Further, experimental approaches have shown that 
mitochondrial genetic variation affects a range of phenotypic traits, including ecologically relevant life history traits (e.g., Rand et al. 2001; Dowling et al. 2008; Ballard \& Melvin 2010; Arnqvist et al. 2010; Houtkooper et al. 2013). Functional mitochondrial haplotype variation should thus rapidly be exhausted by positive or negative selection (Rand et al. 2001). Yet, mtDNA typically exhibits sizeable levels of genetic variation in natural populations (e.g., Clark 1984; Moritz et al. 1987; Nachman et al. 1996). This suggests that additional processes shape the evolution of mtDNA (Bazin et al. 2006).

Current theoretical models, based on positive selection, have suggested that mtDNA polymorphism can only be maintained under special and very restrictive conditions (Clark 1984; Babcock \& Asmussen 1996; Rand et al. 2001; Liu \& Asmussen 2007). In general, the most potent mechanism that can maintain DNA sequence variation is balancing selection by negative frequency-dependent selection (henceforth, NFDS) (Mitchell-Olds et al. 2007), where the relative fitness of an allele or a haplotype decreases if its frequency increases in a population. There are several singular examples of NFDS being important for the maintenance of genetic variation in the nuclear genome (Mitchell-Olds et al. 2007); notably in genes mediating self-incompatibility in plants (Charlesworth 2006), in immunity-related genes such as the major histocompatibility complex in vertebrates (Loisel et al. 2006), in genes affecting behaviour (Fitzpatrick et al. 2007) and for haplotypes/karyotypes with largely unknown function such as inversions (Kojima \& Tobari 1969). In terms of genes coding for ecologically relevant life history characters, however, empirical evidence is limited to phenotypic data alone and theory has had a historical tendency to ignore NFDS (Doebeli \& Ispolativ 2010). With regards to mtDNA, it has been hypothesized that NFDS may often act to maintain mtDNA polymorphism (Gregorius \& Ross 1984), through its association with variation in life 
history traits (Ballard \& Melvin 2010). Although direct experimental tests are lacking (Dowling et al. 2008), a few remarkable observations in insects are consistent with balancing selection by NFDS. First, mtDNA polymorphism in natural fruit fly populations has sometimes been found to be temporally (Adrianov et al. 2008) and spatially (Oliver et al. 2005) stable. Second, observations of temporal stability of mtDNA haplotype frequencies in unreplicated and closed laboratory fruit fly populations (Macrae \& Anderson 1988; Oliver et al. 2005) suggest that NFDS may be operating. Third, widespread sympatric coexistence between distinct and ancient mtDNA haplotype families consistent with NFDS has been observed in, for example, ladybirds (Jiggins \& Tinsley 2005) and moths (Kvie et al. 2013). Here, we use a very large number of laboratory populations of an insect model system (the seed beetle Callosobruchus maculatus) to provide, to our knowledge, the first experimental test of the hypothesis that NFDS acts to maintain mtDNA diversity.

\section{MATERIALS AND METHODS}

Seed beetles of the genus Callosobruchus (Coleoptera, Bruchidae) complete their life cycle within a single seed of their legume hosts and are common cosmopolitan pests on stored legumes. Polymorphism in mtDNA is very pronounced in these seed beetles, both within and between natural populations, and a very large number of haplotypes have been sequenced from natural populations of $C$. maculatus. We tested for NFDS by seeding beetle populations with beetles carrying either of two alterative mtDNA haplotypes at a known frequency. Our primary goal was to test the effect of starting frequency (either common or rare) on the evolutionary fate of a given mtDNA haplotype over many generations. Here, we give only an overview of the materials and methods 
used. A complete description of our experimental procedures is given in the Supporting Information.

\section{Mitonuclear introgression lines}

In order to isolate the genetic effects of mtDNA from the nuclear genome with which it was originally associated, our experiments were based on mtDNA introgression lines. Three distinct mtDNA haplotypes (2-3\% sequence divergence) were expressed in either of three outbred nuclear genetic backgrounds in fully crossed manner (i.e., $3 \times 3$ types of mitonuclear introgression lines, each replicated twice), through repeated introgressive backcrossing for 15 generations. The fact that we thus staged 18 independent introgression lines not only allowed us to assess effects of particular mtDNA haplotypes across several nuclear genetic backgrounds but also to exclude the possibility that capricious line specific genetic effects would influence our results. To preclude the possibility of cytoplasmic bacterial infections, we treated all introgression lines with antibiotics (tetracycline hydrochloride).

\section{Experimental procedure}

We established each of 180 experimental populations with beetles $(N=500)$ representing a mixture of two distinct mitonuclear lines (at a precisely known proportion of 0.2 and 0.8 ) that shared the same nuclear genetic background but differed in their mtDNA haplotype. We employed a fully crossed three-way design in which the factors were mtDNA haplotypes in competition, nuclear genetic background and starting frequency of the focal haplotype $(3 \times 3 \times 2=18$ cells in the design, each replicated 10 times). The two haplotypes were then allowed to compete for 10 generations at a fixed population size $(N=500)$ under laboratory conditions with both thermal and resource 
heterogeneity. At generation 10, we estimated the mtDNA haplotype frequency in each experimental population by sequencing ( $650 \mathrm{bp}$ of cytochrome oxidase subunit I) a random sample of 10 individuals from each population (i.e., $N=1800$ individuals sequenced).

\section{Statistical analyses}

We used the estimated change in haplotype frequency from the start to the end of the experiment in each replicate population $(\Delta f)$ as the response variable in the statistical models reported here. Under the null hypothesis of no selection on mtDNA alleles, the mean value of this parameter equals zero $\left(\mathrm{H}_{0}: \overline{\Delta f}=0\right)$. See Supporting Information for additional and supporting statistical evaluations of our results.

\section{RESULTS}

We asked whether the frequency of a given mitochondrial haplotype increased when initially rare and decreased when common over the course of our experiments and whether frequency changes $(\Delta f)$ were due to the particular mtDNA haplotype pair or to the nuclear genetic background in which haplotype pairs were competing. Whether a given focal haplotype was common or rare at the beginning of the experiment had a highly significant and sizeable effect on the change in haplotype frequency among all of the three mtDNA haplotypes used (Table 1). Overall, the frequency of the rare mtDNA haplotype increased across experimental populations by 5.3\% (95\% CI: $0.027-0.080 ; t$ $=4.0 ; d f=177 ; p<0.001$ ) and, consequently, the frequency of the common haplotype decreased by the same amount (Fig. 1a). Thus, changes in mtDNA haplotype frequencies during the course of the experiment were indeed negatively frequency-dependent. 
Furthermore, the observed negative frequency-dependent effects were consistent over nuclear backgrounds (Fig. 1a) and particular mtDNA haplotypes (Fig. 1b; Table 1).

A closer examination of our results suggests an additional facet of the evolution of mtDNA haplotype frequencies in our experimental populations. The strength of NFDS was, to some extent, contingent upon the interaction between nuclear genetic background and competing mtDNA haplotype, as suggested by significant three-way interactions between starting frequency $\times$ competing mtDNA haplotype $\times$ nuclear genetic background for two out of three focal mtDNA haplotypes (Table 1). The observed effect was apparently caused primarily by NFDS (i.e., the effect of starting frequency) being particularly strong when a focal haplotype was competing against another haplotype within the focal haplotype's own nuclear genetic background (see Supporting Information). For example, the strength of NFDS was stronger when the B haplotype competed against the Y haplotype within a B nuclear genetic background compared with a $C$ nuclear genetic background $(|\Delta f|$ when rare $+|\Delta f|$ when common $=0.29$ and 0.05 , respectively) .

\section{DISCUSSION}

The results of our experiments were remarkably clear-cut: NFDS acted to maintain mtDNA polymorphism within our experimental populations and this pattern was consistent over multiple mtDNA alleles and nuclear genetic backgrounds. Below we briefly discuss four facets of our results. First, we address the likely mechanism behind the observed NFDS. Second, we discuss some of the ramifications of our findings for the use of mtDNA frequency data in population biology and ecology. Third, we note that our results are consistent with a role for mitonuclear epistasis for fitness. Fourth, we suggest 
that our findings may also have wider implications for our understanding of the maintenance of genetic variation in ecologically relevant life history traits.

While several mechanisms could in theory have contributed to the NFDS on mtDNA haplotypes that we observed, we suggest that the general scenario delineated by Lewontin (1974) is by far the most probable one. Lewontin (1974) proposed that NFDS within populations may be pervasive because whenever "a genotype is its own worst enemy, its fitness will decrease as it becomes more common". His proposition was built on three prerequisites, all of which were upheld in our experiment. First, it requires environmental heterogeneity (Kassen 2002). We allowed our experimental populations to evolve under conditions in which they experienced small-scaled environmental heterogeneity both with regards to resource type and thermal conditions. Second, it requires genotype-by-environment interactions, such that the relative performance of different genotypes depends upon the biotic and /or abiotic environment in which they live (Kang \& Gauch 1996). We note that the mtDNA haplotypes used in our experimental populations are known to show complex genotype-by-environment interactions for life history traits, for example temperature specific growth rate (Dowling et al. 2007), metabolic rate (Arnqvist et al. 2010) and the economics of reproduction (Dowling et al. 2010), such that the relative performance of haplotypes differs across environments. Third, it assumes that there is competition for resources. Our experimental populations evolved under conditions of pronounced juvenile resource competition.

There are striking similarities between Lewontin's (1974) proposed scenario and other negative-frequency dependent processes thought to maintain diversity at a range of ecological and evolutionary scales (e.g., Chesson 2000; Kassen 2002). In fact, we suggest 
that Lewontin's (1974) three prerequisites are likely to prevail in many natural populations and NFDS within populations may thus often act to promote the maintenance of mtDNA polymorphism. Environmental heterogeneity is ubiquitous (Kassen 2002) and resource competition is certainly strong in many taxa (Gurevitch et al. 1992). Moreover, genotype-by-environment interactions seem to be a prominent feature of the phenotypic effects of mitochondrial genetic variation (Dowling et al. 2008). The facts that the effects seen in our experiment were largely independent of nuclear genetic background and were consistent across replicate introgression lines also implicate a general mechanism.

Irrespective of mechanism, our demonstration of NFDS on mtDNA haplotypes has important potential implications for studies that use haplotype frequency data to infer population level processes in nature. Based on the assumption that mtDNA variation is selectively neutral (Moritz et al. 1987; Moritz 1994) such data has been extensively used for more than three decades to infer, for example, rates of migration, colonization events, effective population size, gene flow, population structure, phylogeography, introgression in hybrid zones and several other population parameters (Avise et al. 1987; Harrison 1989). Such inferences will be fundamentally problematic if mtDNA haplotype frequencies commonly reflect selection and adaptation rather than withinpopulation coalescence and diffusion of selectively neutral haplotypes across populations (Ballard \& Kreitman 1995; Ballard \& Whitlock 2004; Ballard \& Rand 2005; Bazin et al. 2006; Dowling et al. 2008). This concern is deepened by (i) the fact that that the large scale pattern of variation in mtDNA polymorphism is often inconsistent with the neutrality assumption (e.g., Bazon et al. 2006) and (ii) the fact that experimental tests often reject neutrality (Ballard \& Melvin 2010; Dowling et al. 2008). We note that 
studies based on genetic variation in mtDNA markers which is characterized exclusively by synonymous substitutions or is located in non-coding regions are of course not immune to these potential problems, simply because mtDNA haplotypes may show "hidden" non-synonymous substitutions in sites that have not been sequenced. We suggest that the neutrality assumption of mtDNA variation should be adequately tested in studies that use haplotype frequency data to infer process from pattern (Ballard \& Kreitman 1995; Bazin et al. 2006; Galtier et al. 2009). Unfortunately, however, it is very difficult to devise unambiguous statistical tests for NFDS based on the frequency distribution of mtDNA haplotypes within open natural populations where the demographic history is unknown (using an infinite allele model) (Whittam et al. 1986; Gerber et al. 2001; Jiggins \& Tinsley 2005; Galtier et al. 2009). Yet, our results provide a basis for proposing that NFDS should be tested for in studies using the pattern of mtDNA haplotype frequency data to infer population level processes.

Products of the mitochondrial and the nuclear genome join to form the major energy producing cascade within mitochondria (Ballard \& Melvin 2010) and there are therefore good reasons to believe that mitonuclear epistatic interactions should be important. Mitonuclear epistasis has been experimentally documented for life history traits (Dowling et al. 2008; Ellison \& Burton 2008) and the pattern of mtDNA protein evolution is consistent with a major role for epistasis (Breen et al. 2012). Our study suggests an additional role for mitonuclear epistasis in mtDNA evolution. The fact that the strength of NFDS seemed, to some extent, contingent upon mitonuclear combination suggests that the experimental evolution of mtDNA was affected by the nuclear genome with which it was co-expressed (Fos et al. 1990). This implies that coadaptation between mitochondrial and nuclear genes, which is well documented (Rawson \& Burton 2002; 
Rand et al. 2004; Ellison \& Burton 2008, Houtkooper et al. 2013), may also affect NFDS. Such intergenomic epistasis predict that the ecological and evolutionary dynamics of mtDNA haplotypes may be quite complex (Rand et al. 2001), especially when considering sex-specific effects of mtDNA variation (Innocenti et al. 2011), and NFDS may thus also contribute to the maintenance of nuclear genetic variation. Thus, our results suggests that mtDNA polymorphism in natural populations of our model system represents a complex interaction between NFDS, sex-specific positive/negative selection (Dowling et al. 2007), environmental variation (Arnqvist et al. 2010), mitonuclear epistasis (Dowling et al. 2010), genetic drift and gene flow.

A persistent problem with studying the maintenance of variation in ecologically relevant life history traits lies in relating variation in important life history phenotypes to genetic variation at the loci that control them (Lewontin 1974; Charlesworth \& Hughes 2000; Mitchell-Olds et al. 2007). The fact that many life history traits are influenced by a very large number of genes with small effects (Charlesworth \& Hughes 2000) aggravates this problem. Here, mtDNA markers provide an interesting alternative for the more general study of life history genotypes, simply because they represent haplotypes containing a large set of well characterized and non-recombining genes with important effects on metabolic and life history phenotypes (Ballard and Melvin 2010). Future studies of mitochondrial genes may thus help improve our general understanding of the genetics, ecology and evolution of life histories. To the extent that our results speaks to this more general question, they are certainly consistent with the view that balancing selection though NFDS is an underrated contributor to standing genetic variation in life history traits in natural populations (Doebeli \& Ispolatov 2010). 
Our understanding of the ecological and evolutionary significance of mtDNA is currently being revised (Ruiz-Pesini et al. 2004; Ballard \& Rand 2005; Bazin et al. 2006; Dowling et al. 2008). The traditional view that sequence variation in the mitochondrial genome is selectively neutral is being displaced by a growing body of evidence for selection on mitochondrial genes from a variety of empirical domains and this paradigm shift has wide-ranging evolutionary and ecological implications (Ballard \& Whitlock 2004; Ballard \& Rand 2005; Galtier et al. 2009; Arnqvist et al. 2010). Our study adds a novel and critical piece to our understanding of mtDNA polymorphism by experimentally demonstrating that selection acts upon mtDNA and, most importantly, by showing that NFDS acts to maintain non-neutral mitochondrial genetic variation. Challenges for future research include uncovering the processes that generate NFDS, which should involve a combination of ecological and evolutionary experimental approaches, but also developing methods that may allow us to detect the footprint of NFDS in natural populations.

\section{ACKNOWLEDGEMENTS}

We thank N. Amvrosiadi, M. Avolio, C. Hotzy and E. Gustafsson for assistance during the experimental procedure, Uppsala Genome Centre (SciLifeLab) personnel for their assistance with the sequencing effort, and S. H. Alonzo, D. Dowling, J. Höglund, P. J. Palsbøll and the GENCON lab group for their constructive comments on earlier versions of this manuscript. This work was funded by the European Research Council (AdG294333 to G.A) and the Swedish Research Council (621-2010-5266 to G.A.). 


\section{REFERENCES}

1.

Adrianov, B.V., Sorokina, S., Miuge, N.S., Reznik, N.L. \& Mitrofanov, V.G. (2008). Dynamics of mitochondrial polymorphism in a natural population of Drosophila littoralis. Genetika, 44, 195-201.

2.

Arnqvist, G., Dowling, D.K., Eady, P., Gay, L., Tregenza, T., Tuda, M. \& Hosken, D.J. (2010). Genetic architecture of metabolic rate: environment specific epistasis between mitochondrial and nuclear genes in an insect. Evolution, 64, 3354-3363.

3.

Avise, J.C., Arnold, J., Ball, R.M., Bermingham, E., Lamb, T., Neigel, J.E., Reeb, C.A. \& Saunders, N.C. (1987). Intraspecific phylogeography - the mitochondrial DNA bridge between populaion genetics and systematics. Ann. Rev. Ecol. Evol. Syst., 18, 489-522.

4.

Babcock, C.S. \& Asmussen, M.A. (1996). Effects of differential selection in the sexes on cytonuclear polymorphism and disequilibria. Genetics, 144, 839-853.

5.

Ballard, J.W. \& Melvin, R.G. (2010). Linking the mitochondrial genotype to the organismal phenotype. Mol. Ecol., 19, 1523-1539.

6.

Ballard, J.W. \& Rand, D.M. (2005). The population biology of mitochondrial DNA and its phylogenetic implications. Ann. Rev. Ecol. Evol. Syst., 36, 621-642.

7. 
Ballard, J.W.O. \& Kreitman, M. (1995). Is mitochondrial DNA a strictly neutral marker? Trends Ecol. Evol., 10, 485-488.

8.

Ballard, J.W.O. \& Whitlock, M.C. (2004). The incomplete natural history of mitochondria. Mol. Ecol., 13, 729-744.

9.

Bazin, E., Glemin, S. \& Galtier, N. (2006). Population size does not influence mitochondrial genetic diversity in animals. Science, 312, 570-572.

10.

Breen, M.S., Kemena, C., Vlasov, P.K., Notredame, C. \& Kondrashov, F.A. (2012). Epistasis as the primary factor in molecular evolution. Nature, 490, 535-538.

11.

Charlesworth, B. \& Hughes, K.A. (2000). The maintenance of genetic variation in lifehistory traits. In: Evolutionary Genetics: from Molecules to Morphology, eds. Singh, R.S. \& Krimbas, C.B., Cambridge University Press, Cambridge, UK, pp. 369-392.

12.

Charlesworth, D. (2006). Balancing selection and its effects on sequences in nearby genome regions. PLoS Genetics, 2, e64.

13.

Chesson, P. (2000). Mechanisms of maintenance of species diversity. Ann. Rev. Ecol. Syst., 31, 343-366.

14.

Clark, A.G. (1984). Natural selection with nuclear and cytoplasmic transmission. I. A deterministic model. Genetics, 107, 679-701.

15. 
Doebeli, M. \& Ispolatov, I. (2010). Complexity and diversity. Science, 328, 494-497.

16.

Dowling, D.K., Abiega, K.C. \& Arnqvist, G. (2007). Temperature-specific outcomes of cytoplasmic-nuclear interactions on egg-to-adult development time in seed beetles. Evolution, 61, 194-201.

17.

Dowling, D.K., Friberg, U. \& Lindell, J. (2008). Evolutionary implications of non-neutral mitochondrial genetic variation. Trends Ecol. Evol., 23, 546-554.

18.

Dowling, D.K., Meerupati, T., Arnqvist, G. (2010). Cytonuclear interactions and the economics of mating in seed beetles. Am. Nat., 176, 131-140.

19.

Ellison, C.K. \& Burton, R.S. (2008). Interpopulation hybrid breakdown maps to the mitochondrial genome. Evolution, 62, 631-638.

20.

Fitzpatrick, M.J., Feder, E., Rowe, L. \& Sokolowski, M.B. (2007). Maintaining a behaviour polymorphism by frequency-dependent selection on a single gene. Nature, 447, 210-212.

21.

Fos, M., Dominguez, M.A., Latorre, A. \& Moya, A. (1990). Mitochondrial DNA evolution in experimental populations of Drosophila subobscura. Proc. Natl. Acad. Sci. USA, 87, $4198-4201$.

22. 
Frankham, R. (2012). How closely does genetic diversity in finite populations conform to predictions of neutral theory? Large deficits in regions of low recombination. Heredity, 108, 167-178

23.

Galtier, N., Nabholz, B., Glemin, S. \& Hurst, G.D.D. (2009). Mitochondrial DNA as a marker of molecular diversity: a reappraisal. Mol. Ecol., 18, 4541-4550.

24.

Gerber, A.S., Loggins, R., Kumar, S. \& Dowling, T.E. (2001). Does nonneutral evolution shape observed patterns of DNA variation in animal mitochondrial genomes? Ann. Rev. Genet., 35, 539-566.

25.

Grant, W.S., Spies, I.B. \& Canino, M.F. (2006). Biogeographic evidence for selection on mitochondrial DNA in North Pacific walleye pollock Theragra chalcogramma.J. Hered., 97, 571-580.

26.

Gregorius, H.R. \& Ross, M.D. (1984). Selection with gene-cytoplasm interactions. I. Maintenance of cytoplasm polymorphisms. Genetics, 107, 165-178.

27.

Gurevitch, J., Morrow, L.L., Wallace, A. \& Walsh, J.S. (1992). A meta-analysis of competition in field experiments. Am. Nat., 140, 539-572.

28.

Harrison, R.G. (1989). Animal mitochondrial DNA as a genetic marker in population and evolutionary biology. Trends Ecol. Evol., 4, 6-11.

29. 
Houtkooper, R.K., Mouchiroud, L., Ryu, D., Moullan, N., Katsyuba, E., Knott, G., Williams, R.W. \& Auwerx, J. (2013) Mitonuclear protein imbalance as a conserved longevity mechanism. Nature, 497, 451-457.

30.

Innocenti, P., Morrow, E.H. \& Dowling, D.K. (2011). Experimental evidence supports a sex-specific selective sieve in mitochondrial genome evolution. Science, 332, 845848.

31.

Jiggins, F.M. \& Tinsley, M.C. (2005). An ancient mitochondrial polymorphism in Adalis bipunctata linked to a sex-ratio-distorting bacterium. Genetics, $171,1115-1124$.

32.

Kang, M.S. \& Gauch, H.G. (1996). Genotype-by-Environment Interaction. CRC Press, Boca Raton, Florida.

33.

Kassen, R. (2002). The experimental evolution of specialists, generalists, and the maintenance of diversity. J. Evol. Biol., 15, 173-190.

34.

Kojima, K.I. \& Tobari, Y.N. (1969). Selective modes associated with karyotypes in Drosophila ananassae. II. Heterosis and frequency-dependent selection. Genetics, 63, $639-651$.

35.

Kvie, K.S., Hogner, S., Aarvik, L., Lifjeld, J.T. \& Johnsen, A. (2013). Deep sympatric mtDNA divergence in the autumnal moth (Epirrita autumnata). Ecol. Evol., 3, 126-144.

36. 
Lewontin, R.C. (1974). The Genetic Basis of Evolutionary Change. Columbia University Press, New York.

37.

Liu, R. \& Asmussen, M.A. (2007). Cytonuclear dynamics in selfing populations under selection. Theor. Pop. Biol., 71, 445-453.

38.

Loisel, D.A., Rockman, M.V., Wray, G.A., Altmann, J. \& Alberts, S.C. (2006). Ancient polymorphism and functional variation in the primate MHC-DQA1 5 ' cis-regulatory region. Proc. Natl. Acad. Sci. USA, 103, 16331-16336.

39.

Macrae, A.F. \& Anderson, W.W. (1988). Evidence for non-neutrality of mitochondrial DNA haplotypes in Drosophila pseudoobscura. Genetics, 120, 485-494.

40.

Mitchell-Olds, T., Willis, J.H. \& Goldstein, D.B. (2007). Which evolutionary processes influence natural genetic variation for phenotypic traits? Nature Rev. Genet., 8, 845856.

41.

Moritz, C. (1994). Applications of mitochondrial DNA analysis in conservation - a critical review. Mol. Ecol., 3, 401-411.

42.

Moritz, C., Dowling, T.E. \& Brown, W.M. (1987). Evolution of animal mitochondrial DNA relevance for population biology and systematics. Ann. Rev. Ecol. Syst., 18, 269-292. 43.

Nachman, M.W., Brown, W.M., Stoneking, M. \& Aquadro, C.F. (1996). Nonneutral mitochondrial DNA variation in humans and chimpanzees. Genetics, 142, 953-963. 
44 .

Oliver, P., Balanya, J., Ramon, M., Picornell, A., Serra, L., Moya, A. \& Castro, J.A. (2005). Population dynamics of the 2 major mitochondrial DNA haplotypes in experimental populations of Drosophila subobscura. Genome, 48, 1010-1018.

45.

Rand, D.M., Clark, A.G. \& Kann, L.M. (2001). Sexually antagonistic cytonuclear fitness interactions in Drosophila melanogaster. Genetics, 159, 173-187.

46.

Rand, D.M., Haney, R.A. \& Fry, A.J. (2004). Cytonuclear coevolution: the genomics of cooperation. Trends Ecol. Evol., 19, 645-653.

47.

Rawson, P.D. \& Burton, R.S. (2002) Functional coadaptation between cytochrome c and cytochrome c oxidase within allopatric populations of a marine copepod. . Proc. Natl. Acad. Sci. USA, 99, 12955-12958.

48.

Ruiz-Pesini, E., Mishmar, D., Brandon, M., Procaccio, V. \& Wallace, D.C. (2004). Effects of purifying and adaptive selection on regional variation in human mtDNA. Science $303,223-226$.

49.

Whittam, T.S., Clark, A.G., Stoneking, M., Cann, R.L. \& Wilson, A.C. (1986). Allelic variation in human mitochondrial genes based on patterns of restriction site polymorphism. Proc. Natl. Acad. Sci. USA, 83, 9611-9615.

50. 
Willett, C.S. \& Burton, R.S. (2003). Environmental influences on epistatic interactions: viabilities of cytochrome c genotypes in interpopulation crosses. Evolution, 57, 2286-2292.

\section{SUPPORTING INFORMATION}

Additional Supporting Information may be downloaded via the online version of this article at Wiley Online Library (www.ecologyletters.com). 


\section{FIGURE LEGENDS}

Figure 1 Overall, mtDNA haplotypes decreased in frequency when common and increased in frequency when rare across all three nuclear genetic backgrounds. Here, each pair of matched lines (one in the top part of the graph and one in the mirroring bottom part) represents the mean haplotype frequencies (over 60 replicate populations) in one of the three distinct nuclear genetic backgrounds (black circle: C; red triangle: B; blue diamond: Y). Error bars represent SEM. Note scale break along the ordinate.

Figure 2 A given mtDNA haplotype decreased in frequency when initially common and increased in frequency when initially rare. Shown here are mean changes in haplotype frequency from the start to the end of the experiment $(\Delta f)$ for the different focal mtDNA haplotypes (black circle: C; red triangle: B; blue diamond: Y) and starting frequencies. Error bars represent 95\% bootstrap CI, based on 10000 bootstrap replicates, and asterisks indicate significant $(P<0.05)$ deviations from zero (one sample $t$-tests of $\mathrm{H}_{0}: \Delta f$ $=0 ; d f=59$ ). Note that the variation in haplotype frequency changes across replicate populations was symmetrical and of similar magnitude across haplotypes and starting frequencies. Combined probability tests showed that, across all three haplotypes, $\Delta f<0$ when a focal haplotype was common $(p<0.001)$ while $\Delta f>0$ when a focal haplotype was rare $(p<0.001)$ (see Supporting Information). 
Table 1 The effect of starting frequency on the change in mtDNA haplotype frequencies

\begin{tabular}{lcccccccccc}
\hline Focal mtDNA haplotype: & \multicolumn{3}{c}{ C } & \multicolumn{9}{c}{ B } & \multicolumn{3}{c}{ Y } \\
\hline Source & $d f$ & $F$ & $p$ & $d f$ & $F$ & $p$ & $d f$ & $F$ & $P$ \\
\hline Starting frequency (SF) & $1,98.7$ & 11.64 & $<\mathbf{0 . 0 0 1}$ & $1,98.4$ & 7.25 & $\mathbf{0 . 0 0 8}$ & $1,98.6$ & 18.65 & $<\mathbf{0 . 0 0 1}$ \\
Competing haplotype (CH) & $1,5.9$ & 0.03 & 0.875 & $1,6.2$ & 0.49 & 0.510 & $1,6.0$ & 0.25 & 0.635 \\
Nuclear background (NB) & $2,3.8$ & 0.09 & 0.916 & $2,2.2$ & 4.49 & 0.170 & $2,4.1$ & 0.99 & 0.444 \\
SF $\times$ CH & $1,98.7$ & 2.69 & 0.104 & $1,98.4$ & 1.01 & 0.316 & $1,98.6$ & 0.32 & 0.570 \\
SF $\times$ NB & $2,98.7$ & 0.75 & 0.473 & $2,98.4$ & 1.13 & 0.328 & $2,98.6$ & 1.01 & 0.368 \\
CH $\times$ NB & $2,5.9$ & 0.51 & 0.627 & $2,6.2$ & 0.52 & 0.620 & $2,6.0$ & 1.19 & 0.366 \\
SF $\times$ CH $\times$ NB & $2,98.7$ & 0.29 & 0.752 & $2,98.4$ & 3.28 & $\mathbf{0 . 0 4 1}$ & $2,98.6$ & 3.29 & $\mathbf{0 . 0 4 0}$ \\
\hline
\end{tabular}

Linear mixed models (REML estimation) of the effects of fixed factorial variables on changes in haplotype frequency $(\Delta f)$ during the course of the experiment, when modelled as $\Delta f$ across all replicates that involved a given focal mtDNA haplotype $(n=$ 120 for all three models). In all models, introgression line identities were included as random effects variables. 
Table 1. The effect of starting frequency on the change in mtDNA haplotype frequencies.

\begin{tabular}{l|rrr|rrr|rrr}
\hline Focal mtDNA haplotype: & \multicolumn{3}{|c}{ C } & \multicolumn{3}{|c|}{ B } & \multicolumn{3}{|c}{ Y } \\
\hline Source & $d f$ & $F$ & $p$ & $d f$ & $F$ & $p$ & $d f$ & $F$ & $P$ \\
\hline Starting frequency (SF) & $1,98.7$ & 11.64 & $<\mathbf{0 . 0 0 1}$ & $1,98.4$ & 7.25 & $\mathbf{0 . 0 0 8}$ & $1,98.6$ & 18.65 & $<\mathbf{0 . 0 0 1}$ \\
Competing haplotype (CH) & $1,5.9$ & 0.03 & 0.875 & $1,6.2$ & 0.49 & 0.510 & $1,6.0$ & 0.25 & 0.635 \\
Nuclear background (NB) & $2,3.8$ & 0.09 & 0.916 & $2,2.2$ & 4.49 & 0.170 & $2,4.1$ & 0.99 & 0.444 \\
SF $\times$ CH & $1,98.7$ & 2.69 & 0.104 & $1,98.4$ & 1.01 & 0.316 & $1,98.6$ & 0.32 & 0.570 \\
SF $\times$ NB & $2,98.7$ & 0.75 & 0.473 & $2,98.4$ & 1.13 & 0.328 & $2,98.6$ & 1.01 & 0.368 \\
CH $\times$ NB & $2,5.9$ & 0.51 & 0.627 & $2,6.2$ & 0.52 & 0.620 & $2,6.0$ & 1.19 & 0.366 \\
SF $\times$ CH $\times$ NB & $2,98.7$ & 0.29 & 0.752 & $2,98.4$ & 3.28 & $\mathbf{0 . 0 4 1}$ & $2,98.6$ & 3.29 & $\mathbf{0 . 0 4 0}$ \\
\hline
\end{tabular}

Linear mixed models (REML estimation) of the effects of fixed factorial variables on changes in haplotype frequency $(\Delta f)$ during the course of the experiment, when modelled as $\Delta f$ across all replicates that involved a given focal mtDNA haplotype $(n=120$ for all three models). In all models, introgression line identities were included as random effects variables (see Text S1). 


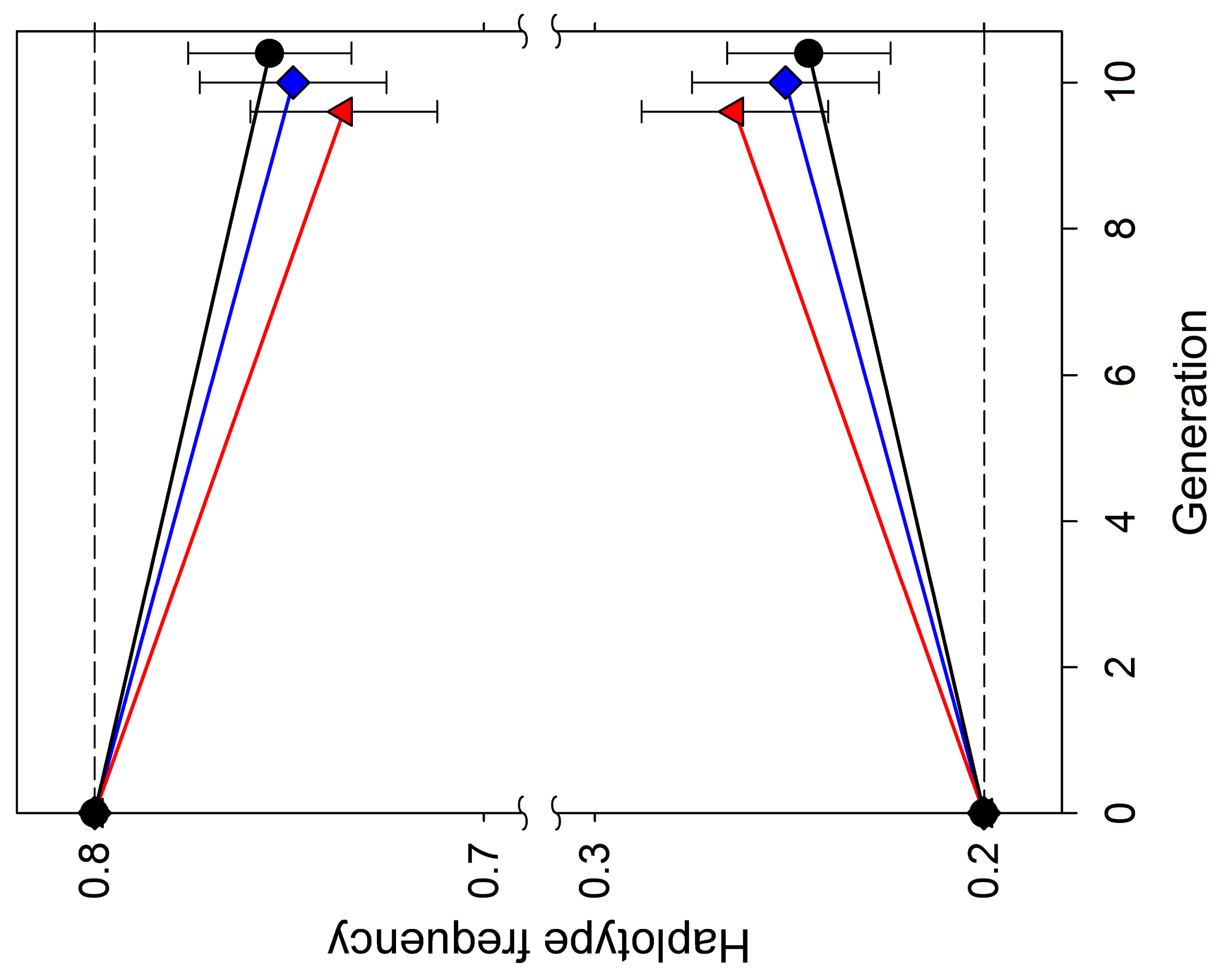




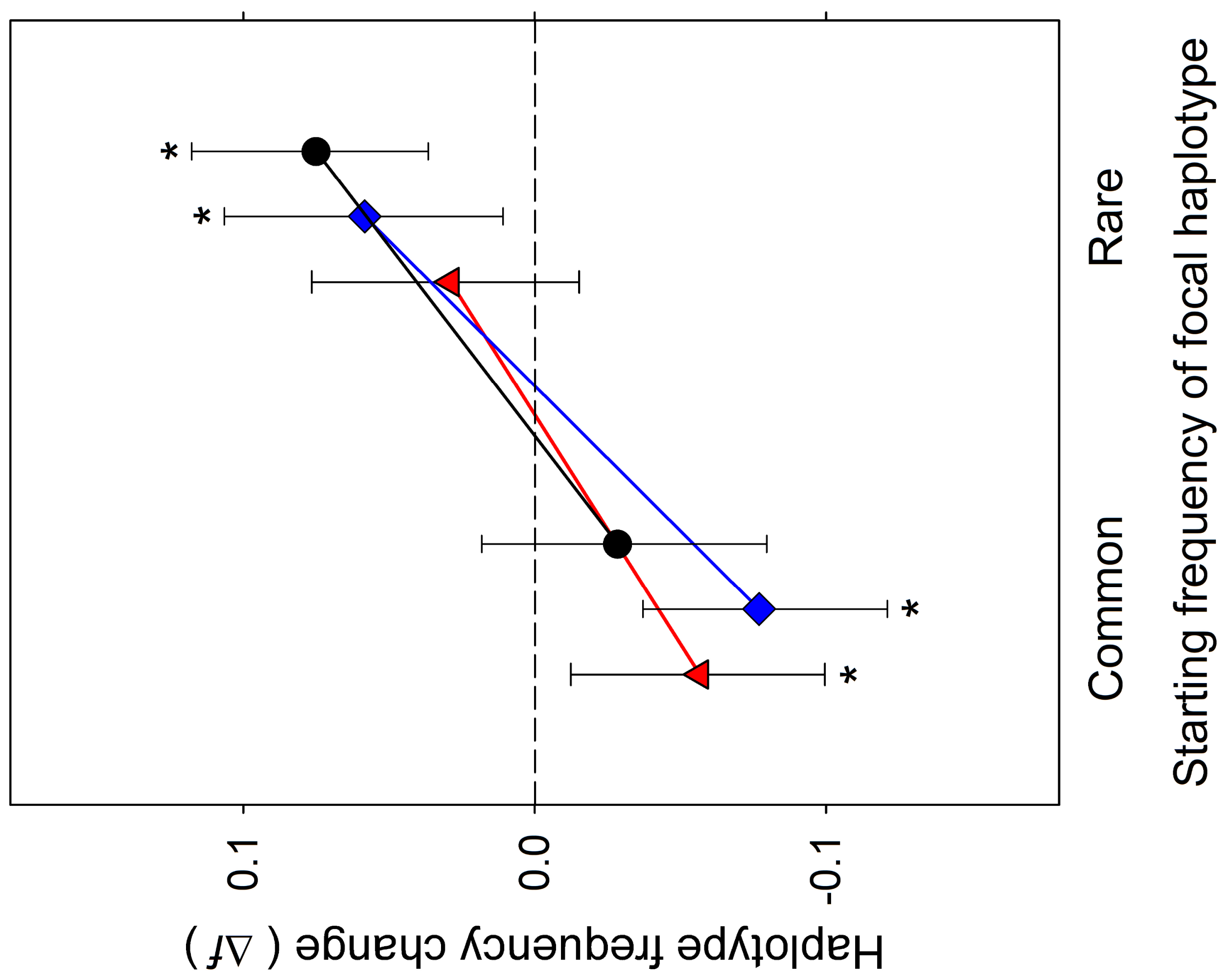

